\title{
Dénombrement des germes totaux et des bactéries coliformes des crèmes consommées dans la région de Téhéran et détermination du taux de contamination de ces crèmes en staphylocoques coagulase positive
}

\author{
par \\ M. KOHNECHAHRI, M. MALEKI, A. FARKHONDEH \\ et R. GHAZVINIAN \\ Département d'Hygiène Alimentaire de l'Université de Téhéran
}

Dans la région de Téhéran, la consommation des crèmes crues et des crèmes dites « chauffées » est beaucoup plus importante que celle des crèmes pasteurisées. La crème crue est en général vendue en gros dans quelques grandes crémeries qui produisent elles-mêmes du lait tandis que la crème dite « chauffée » est en vente chez presque tous les détaillants.

La crème dite " chauffée » est une crème qui est thermo-traitée selon un procédé suggéré par les services d'hygiène publique en vue de résoudre le problème de l'insuffisance des moyens de pasteurisation de la crème de consommation. Il consiste à chauffer la crème crue pendant environ $10 \mathrm{mn}$ à une température voisine de $80^{\circ} \mathrm{C}$. La crème est mise dans une cuve en cuivre étamé et chauffée dans un bain d'eau bouillante en ayant soin d'agiter constamment la crème pendant le chauffage.

Il est évident qu'un tel traitement thermique n'est pas aussi efficace que la pasteurisation industrielle mais dans les conditions actuelles et jusqu'au jour où la pasteurisation de la crème de consommation sera généralisée, l'usage de cette méthode peut éviter considérablement la contamination de la crème.

En tenant compte de l'importance que peut avoir la crème, crue ou chauffée, dans la consommation des produits laitiers et pour faire suite à notre travail précédent [1] nous avons pensé utile de faire une étude sur la numération des germes totaux et des bactéries coliformes des crèmes consommées dans la région de Téhéran en déterminant le taux de contamination de ces crèmes en staphylocoques coagulase positive et évaluer l'efficacité du traitement thermique utilisé pour l'amélioration de la qualité de la crème. 


\section{METHODE ET MATERIEL}

Au cours d'une période entre le 5 avril et la fin du mois de novembre 1971, nous avons prélevé au total 322 échantillons de crème (117 crèmes crues et 205 crèmes chauffées) des crémeries et des magasins de vente de la région de Téhéran. Considérant le niveau d'hygiène dans les différentes parties de la région, un échantillon est prélevé de chacun des lieux de préparation et de vente des crèmes, pris au hasard, en prenant toutes les mesures nécessaires d'asepsie. Les flacons stériles contenant les échantillons sont gardés dans la glace et transportés le plus rapidement possible au laboratoire, puis immédiatement analysés.

Chaque échantillon est utilisé pour l'ensemencement en vue de la numération totale, dénombrement des bactéries coliformes et des staphylocoques coagulase positive. Les milieux suivants sont utilisés :

- milieu de référence [2] pour la numération des germes totaux,

- violet red bile agar [3] et gélose lactosée au désoxycholate [4].

- milieu Baird-Parker [5, 6, 7] pour la recherche des staphylocoques.

La température et la durée d'incubation étaient pour la numération totale de $30^{\circ} \mathrm{C} / 72 \mathrm{~h}$, pour le dénombrement des bactéries coliformes de $37^{\circ} \mathrm{C} / 24 \mathrm{~h}$ et pour la recherche des staphylocoques de $37^{\circ} \mathrm{C} / 24$ et $48 \mathrm{~h}$. Les colonies de staphylocoques sont isolées et ensemencées sur le milieu trypticase soy agar BBL en vue de la recherche ultrieure de la coagulase et dans le cas où le test de la coagulase était positif, le typage de bactériophage était réalisé [8].

\section{RESULTATS}

Le tableau 1 montre les résultats de la numération des germes totaux des crèmes crues et chauffées.

D'après ce tableau, le nombre moyen des germes totaux dans les crèmes crues est beaucoup plus élevé que dans les crèmes chauffées.

Le tableau 2 montre les résultats du dénombrement des bactéries coliformes des crèmes crues et chauffées.

On voit également dans ce tableau la différence de la contamination dans les deux catégories de crèmes.

Le tableau 3 montre les résultats de la détermination du taux de contamination des crèmes en staphylocoques coagulase positive.

D'après ce tableau, le résultat de la recherche des staphylocoques coagulase positive est négatif dans tous les échantillons des crèmes chauffées tandis qu'il est positif dans 5 p. 100 des échantillons de crèmes crues. 
Le tableau 4 montre le pourcentage des types bactériophagiques des staphylocoques isolés des crèmes crues et chauffées.

TABLEAU 1

\begin{tabular}{c|c|c|c|c}
\hline Crèmes & $\begin{array}{c}\text { Nombre } \\
\text { d'examens }\end{array}$ & $\begin{array}{c}\text { Cas } \\
\text { positifs }\end{array}$ & $\begin{array}{c}\text { p. 100 des cas } \\
\text { positifs }\end{array}$ & Moyenne \\
\cline { 1 - 2 } Crues & 117 & 117 & 100 & $35,000,000$ \\
Chauffées & 205 & 191 & 93 & $3,800,000$ \\
\hline
\end{tabular}

TABLEAU 2

\begin{tabular}{c|c|c|c|c}
\hline Crèmes & $\begin{array}{c}\text { Nombre } \\
\text { d'examens }\end{array}$ & $\begin{array}{c}\text { Cas } \\
\text { positifs }\end{array}$ & $\begin{array}{c}\text { p.100 des cas } \\
\text { positifs }\end{array}$ & Moyenne \\
\cline { 2 - 3 } Crues & 117 & 117 & 100 & $1,400,000$ \\
Chauffées & 205 & 132 & 64,4 & 14,250 \\
\hline
\end{tabular}

TABLEAU 3

\begin{tabular}{c|c|c|c|c}
\hline Crèmes & $\begin{array}{c}\text { Nombre } \\
\text { d'examens }\end{array}$ & $\begin{array}{c}\text { Cas } \\
\text { positifs }\end{array}$ & $\begin{array}{c}\text { p. } 100 \text { des cas } \\
\text { positifs }\end{array}$ & Moyenne \\
\cline { 1 - 2 } Crues & 117 & 6 & 5 & 2600 \\
Chautfée & 205 & - & - & - \\
\hline
\end{tabular}

TABLEAU 4

\begin{tabular}{l|c|c}
\hline \multicolumn{1}{c|}{ Groupes } & Nombres des souches & p. 100 \\
\cline { 1 - 2 } Groupe I & & \\
$\quad 79$ & 1 & 16,6 \\
Groupe II & 2 & 33,3 \\
3A/3C & 1 & 16,6 \\
Groupe III & 0 & 0 \\
$\quad 54$ & 0 & 0 \\
Groupe IV & 0 & 0 \\
Groupe mixte & 2 & 33,3 \\
Types divers & 6 & \\
Non typables & & \\
Souches totales & & \\
\hline
\end{tabular}




\section{COMMENTAIRES}

Comme on le voit dans le tableau 1, le traitement thermique, par le procédé mentionné, réduit considérablement les contaminations microbiennes. En ce qui concerne les bactéries coliformes, la crème crue est beaucoup plus contaminée que la crème chauffée cela certainement à cause des faibles mesures d'hygiène prise au stade de la production. La contamination de la crème chauffée (1 p. 100 environ de celle de la crème crue) semble être due aux manipulations diverses pendant le transport et la vente. Quant à la contamination en staphylocoques coagulase positive, la crème chauffée ne contient pas de ces germes et 5 p. 100 seulement des échantillons de crème crue sont contaminés; d'ailleurs la moyenne de la contamination en staphylocoques coagulase positive n'excède pas $2600 \mathrm{ml}$. C'est, sans doute, à cause du taux relativement bas de ces germes dans les laits crus produits dans la région et la grande teneur de ces laits en micro-organismes divers et plus particulièrement bactéries lactiques [1].

En ce qui concerne les types bactériophagiques des staphylocoques isolés, on voit dans le tableau 4 qu'ils sont situés dans des groupes autres que le groupe IV. En tenant compte que ce groupe est constitué, d'après certains auteurs [9], de souches d'origine bovine, nous pouvons conclure que les staphylocoques trouvés dans les crèmes examinées n'ont pas une origine animale et viennent de la contamination par manipulations du lait et de la crème au cours de la production et de la distribution.

\section{S u $\mathbf{m} \mathbf{m}$ a r y}

322 samples of raw and so called " heated " cream produced in Teheran area have been studied for total and coli count with special reference to coagulase positive staphylococci. According to the results obtained, only in 5 p. 100 of raw marked cream we have found coagulase positive staphylococci. No coagulase positive staphylococci has been found in " heated » cream.

\section{Bibliographie}

[1] Ghazvinian (R.), Farkhondeh (A.), Maleki (M.) et Kohnechahri (M.) (1972). Dénombrement des germes totaux et des bactéries coliformes des laits crus délivrés à la centrale laitière de Téhéran, et détermination du taux de contamination de ces laits en staphylocoques coagulase positive. Le Lait, janvier-février, $\mathrm{n}^{\circ}$ 511-512. 
[2] Colony count of liquid milk and dried milk. International Standard, FIL! $I D F, 3,1958$.

[3] Méthode de routine normalisée pour le dénombrement des bactéries coliformes dans le lait cru. Norme Internationale FIL/IDF, 39, 1966.

[4] American Public Health Association. Standard methods for the examination of dairy products, 11th ed., New-York, 1960.

[5] Baird Parker (A. C.) (1962). - J. Appl. Bact., 25, 12.

[6] Thieulin (G.), Basille (D.), Pantaléon (J.), Rosset (R.), Gandon (Y.) et Petit (A.) (1966). - Recherche des staphylocoques pathogènes dans le lait et les produits laitiers. Le Lait, $\mathrm{n}^{\circ} 453-454,131$.

[7] AsPerger (H.) (1965). - Zur Klassifikation der Mikrokokken-Staphylokokken aus milchwirtschaftlicher Sicht. Die Osterreichische Milchwirtschaft. Beilage, 3.

[8] Blair (J. E.) and Williams (R. E. O.) (1961). - Phage typing of staphylococci. Bull. of the World Health Organization, 24, 771.

[9] Munch-Petersen (E.) (1963). - Staphylococci in Food and Food Intoxication. A review and an Applied of Phage typing results. J. Food Sci., vol. $26, \mathrm{n}^{\circ} 6$. 